\title{
An Additional Component Blurring the Transition between Galactic and Extragalactic Cosmic Rays?
}

\author{
Olivier Deligny* \\ CNRS/IN2P3 - IPN Orsay, France \\ E-mail: deligny@ipno.in2p3.fr \\ Imen Al Samarai \\ CNRS/IN2P3 - LPNHE, France \\ E-mail: ialsamar@lpnhe.in2p3.fr
}

\begin{abstract}
Recent KASCADE-Grande and Auger results between $10^{17} \mathrm{eV}$ and $5 \times 10^{18} \mathrm{eV}$ have revealed complex features in the energy spectrum, be it in the all-particle one or in the compositionsensitive ones. They have also revealed that the mass composition is dominated by iron nuclei around $10^{17} \mathrm{eV}$, and by light and intermediate-nuclei elements above $10^{18} \mathrm{eV}$. In this contribution, we argue that these results can be interpreted in a coherent way as the manifestation of an additional component of a different origin from the one responsible for the bulk of Galactic cosmic rays. This component, sub-dominant below $10^{17} \mathrm{eV}$, appears dominant together with the extragalactic one once the standard Galactic component is extinguished above few $10^{17} \mathrm{eV}$, and is responsible for the ankle feature through its rapid suppression above $10^{18} \mathrm{eV}$. Possible signatures left in the large-scale structure of arrival directions are discussed.
\end{abstract}

The 34th International Cosmic Ray Conference,

30 July- 6 August, 2015

The Hague, The Netherlands

${ }^{*}$ Speaker. 
Introduction. Ultra-High Energy Cosmic Rays (UHECRs) are presumably of extragalactic origin. The energy at which these extragalactic CRs start to dominate the overall flux is still an open issue to be deciphered. Since its discovery in 1963 at Volcano Ranch [1], the ankle, a hardening in the all-particle energy spectrum located at $\simeq 5 \times 10^{18} \mathrm{eV}$ [2], has been commonly considered as the natural onset in the energy spectrum marking the transition between only two components of CRs: the Galactic one and the extragalactic one. The aim of this contribution is to put into perspective the tensions between this scenario and recent observations between $10^{17} \mathrm{eV}$ and $\simeq 5 \times 10^{18} \mathrm{eV}$, and to discuss the necessity to introduce a third component for fitting these observations.

Supernova remnants are generally considered as the best candidates that emit the bulk of Galactic Cosmic Rays (GCRs). This is mainly because the intensity of cosmic rays observed on Earth can be produced using $\simeq 10 \%$ of the energetics of these astrophysical objects on the one hand [3]; and the diffusive shock acceleration has been shown to be a mechanism able to convert kinetic energy of the expanding supernova blast wave into accelerated particles on the other hand [4]. Particles are expected to be accelerated following a power-law energy spectrum up to a maximum acceleration energy. Above this energy, the efficiency loss of the acceleration mechanism induces a smooth suppression of the energy spectrum of the accelerated particles. The rigidity-dependent maximum acceleration energy is expected to be of the order of $10^{17} \mathrm{eV}$ for iron nuclei [5]. The highest energy GCRs are thus expected to be smoothly suppressed above this energy. This paradigm of rigidity-dependent acceleration at the sources is supported by the observed knees in the energy spectrum, and in particular by the iron knee located at $\simeq 9 \times 10^{16} \mathrm{eV}[6,7]$. More generally, the energy spectrum, the chemical composition and the anisotropy amplitudes of CRs up to $\simeq 10^{17} \mathrm{eV}$ can be reproduced in a satisfactory way within this paradigm for a diffusion coefficient scaling with energy as $E^{1 / 3}$, as expected for the turbulent component of the Galactic magnetic field described by a Kolmogorov power spectrum [8]. The key point to maintain the anisotropy amplitudes at the level of the observations within the diffusion scenario is to account for the spatial and temporal stochastic distributions of the supernova remnants: in some specific realisations of this scenario, the global dipole amplitude can remain below $1 \%$ even around $10^{17} \mathrm{eV}$ through a subtle interplay of individual stronger dipole amplitudes pertaining to the most recent sources but canceling each other due to opposite phases [8].

The loss of efficiency to accelerate iron nuclei above the iron-knee energy is thus expected to produce a cutoff in the energy spectrum of GCRs above few $10^{17} \mathrm{eV}$. From the observational point of view, recent analyses of the distributions of shower maxima observed at the Pierre Auger Observatory have provided an important element in favor of this scenario [9]. Indeed, between $\simeq 10^{18} \mathrm{eV}$ and the ankle energy, only a mixture of mainly protons and CNO elements provide a reasonable fit of the data. The absence of iron nuclei is striking, since in the scenario of the ankle marking the transition between GCRs and extragalactic ones, these nuclei should compose at least $\simeq 50 \%$ of the flux at $\simeq 10^{18} \mathrm{eV}$.

The transition between GCRs and extragalactic ones could thus occur below the ankle energy. Understanding how this transition is taking place is of central importance, since it would allow us to understand the highest-energy accelerators in the Galaxy. Recently, energy spectra have been inferred for light (that is, protons and He nuclei) and heavy (that is, from CNO to Fe nuclei) elements at the KASCADE-Grande experiment [10]. The softening feature (iron knee) at $\simeq 9 \times$ 
$10^{16} \mathrm{eV}$ can be unambiguously attributed to the heavier elements. Interestingly, a hardening is observed at $\simeq 2 \times 10^{17} \mathrm{eV}$ in the energy spectrum of the light elements. This hardening could be the manifestation of the beginning of the transition between GCRs and extragalactic ones. In this case, however, the origin of the ankle is left unexplained. The aim of this contribution is to argue that the only way to reconcile all observations is to introduce a third component in a similar spirit to the 'component B' discussed in [11] and [12, 13], presumably of Galactic origin, and dominated by CNO elements around $\simeq 10^{18} \mathrm{eV}$.

Extragalactic cosmic rays at ultra-high energies. In a time-honored picture, UHECRs are thought to be accelerated in identical extragalactic sources distributed in the whole Universe. A rigidity-dependent acceleration mechanism at the sources has recently emerged to fit the fact that the mass composition observed at the Pierre Auger Observatory is getting in average heavier between $\simeq 10^{18}$ and $\simeq 3 \times 10^{19} \mathrm{eV}$. With no evolutionary effects that could change their luminosity as a function of the redshift, the injection flux of each source for each mass $i$ is thus assumed to be

$$
J_{i}^{g}(E)=f_{i}^{g} J_{0}^{g}\left(\frac{E}{10^{18} \mathrm{eV}}\right)^{-\gamma_{i}^{g}} f\left(E, E_{\max }^{g}\left(Z_{i}\right)\right)
$$

where $f_{i}^{g}$ is the relative abundance of mass $i, Z_{i}$ the corresponding charge, $J_{0}^{g}$ is the flux normalisation of the source, and $\gamma_{i}^{g}$ the spectral index which might be different between protons and other nuclei depending on the source environment. The function $f$ is a cut-off function, whose precise details are here not relevant. The important point is that the maximum acceleration energy is assumed to be proportional to the charge of the CRs:

$$
E_{\text {max }}^{g}\left(Z_{i}\right)=Z_{i} E_{\max }^{g}(p)
$$

The interactions of CRs with extragalactic background photons are known to modify the injection fluxes. The expectations for the fluxes $J_{i}(E)$ as observed on Earth are thus not identical to the fluxes $J_{i}^{g}(E)$ generated at the sources. Unknown parameters characterising the relative abundances and the spectral index(es) at the sources can be obtained by fitting simultaneously the propagated fluxes $J_{i}(E)$ to the observed all-particle energy spectrum and relative abundances above the ankle energy.

From complete simulation codes $[14,15,16]$, the propagated fluxes for each species roughly appear as power laws that exponentially cut-off at some $E_{\max }$ energies:

$$
J_{i}(E)=f_{i} J_{0}\left(\frac{E}{10^{18} \mathrm{eV}}\right)^{-\gamma_{i}} \exp \left(-E / E_{\max }\left(Z_{i}, A_{i}\right)\right) .
$$

The additional dependence of the propagated $E_{\max }$ with the nuclear mass $A_{i}$ comes from eventual propagation effects related to photo-disintegration processes. Using these simple parameterisations, the relative abundances $f_{i}$ obtained within the extragalactic scenario and that resemble the most to the observed ones above $\simeq 5 \times 10^{18} \mathrm{eV}$ reported in [9] are shown in the left panel of figure 1 . Although more quantitative works should be performed to provide better fits to the data, this simplified approach to obtain the relative abundances $f_{i}$ are sufficient to infer important implications on the origin of CRs around the ankle energy. 

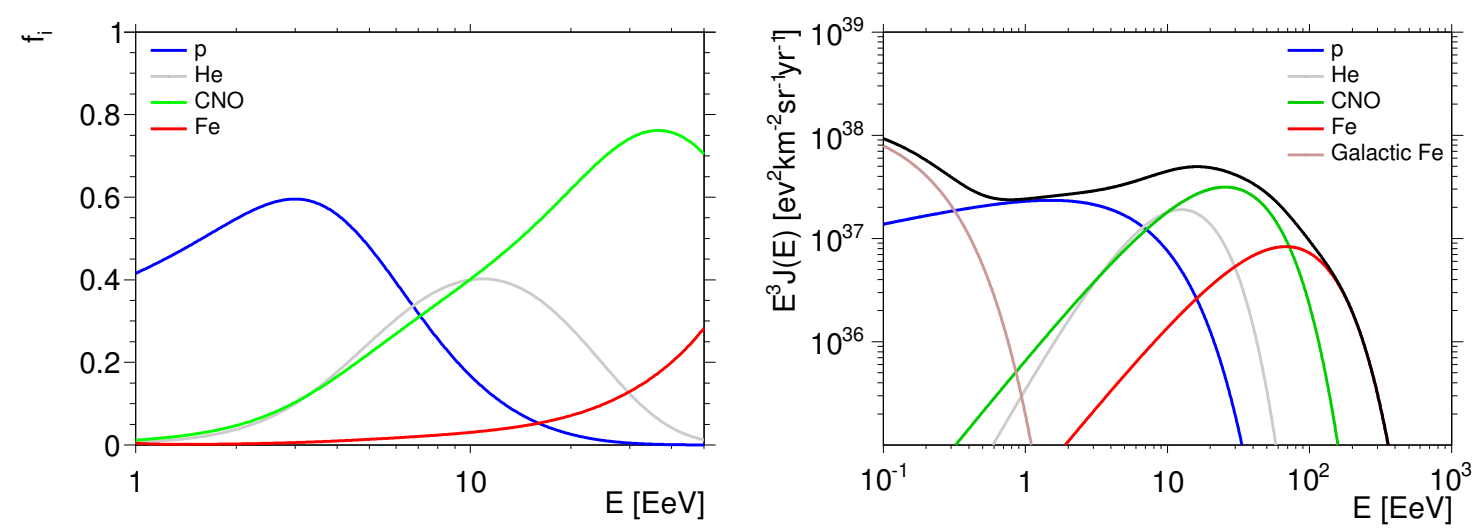

Figure 1: Left: Fraction of different mass group elements expected on Earth in the extragalactic scenario. Right: Natural model of the all-particle energy spectrum above $10^{17} \mathrm{eV}$, obtained as the sum of the end of GCRs composed of Fe elements ('Galactic Fe') and of the propagated extragalactic CRs.

The 'natural' transition. The extragalactic scenario presented just above provides a framework to describe the relative abundances of each species and the all-particle energy spectrum above $5 \times 10^{18} \mathrm{eV}$. Below this energy, it appears from figure 1 that the extragalactic protons have a significant contribution to the all-particle flux. In the following, the normalisation of this extragalactic proton component is fixed at $2 \times 10^{17} \mathrm{eV}$ to the value derived from the KASCADE-Grande report [10], assuming the component referred to as "light" in this report is composed exclusively of protons (that is, no helium nuclei) ${ }^{1}$. To respect the absence of iron nuclei around and above $10^{18} \mathrm{eV}$, the end of GCRs, assumed to be composed of iron nuclei accordingly to the scenario of rigidity-dependent maximum acceleration energy, has to be exponentially suppressed above the iron-knee energy. The different fluxes $J_{i}(E)$ as well as the overall energy spectrum resulting from this global scenario are shown in the right panel of figure 1. As expected, an ankle feature is produced as the consequence of the crossover between a hard power law and a softer one.

The all-particle spectrum depicted in this figure is actually the most natural one in the framework of the ankle marking the transition between the bulk of GCRs and extragalactic ones. In this picture, the ankle is observed to be a smooth change of slope occurring over a half-decade in energy around $10^{18} \mathrm{eV}$. This is clearly in strong tension with the observed features of the actual ankle: a sharp change of slope around $5 \times 10^{18} \mathrm{eV}$. Hence, to reproduce in a satisfactory way the all-particle energy spectrum and in particular the actual ankle feature, an additional ingredient is needed. A precious clue is given in the observed mass composition as reported in [9].

An additional component. The analyses of the $X_{\max }$ distributions in terms of mass composition by means of the EPOS-LHC hadronic interaction model, as reported in [9], have revealed three important features around $10^{18} \mathrm{eV}$ : the quasi-absence of iron nuclei, the presence of $\simeq 55 \%$ of protons, and the presence of $\simeq 40 \%$ of intermediate CNO elements. The presence of these elements

\footnotetext{
${ }^{1}$ This fixed normalisation for the flux of the proton component translates into a harder spectral index for the protons than for the other species after propagation. It is worth noting that a quantitative examination of this point could have in itself important consequences for constraining the source environments [17].
} 

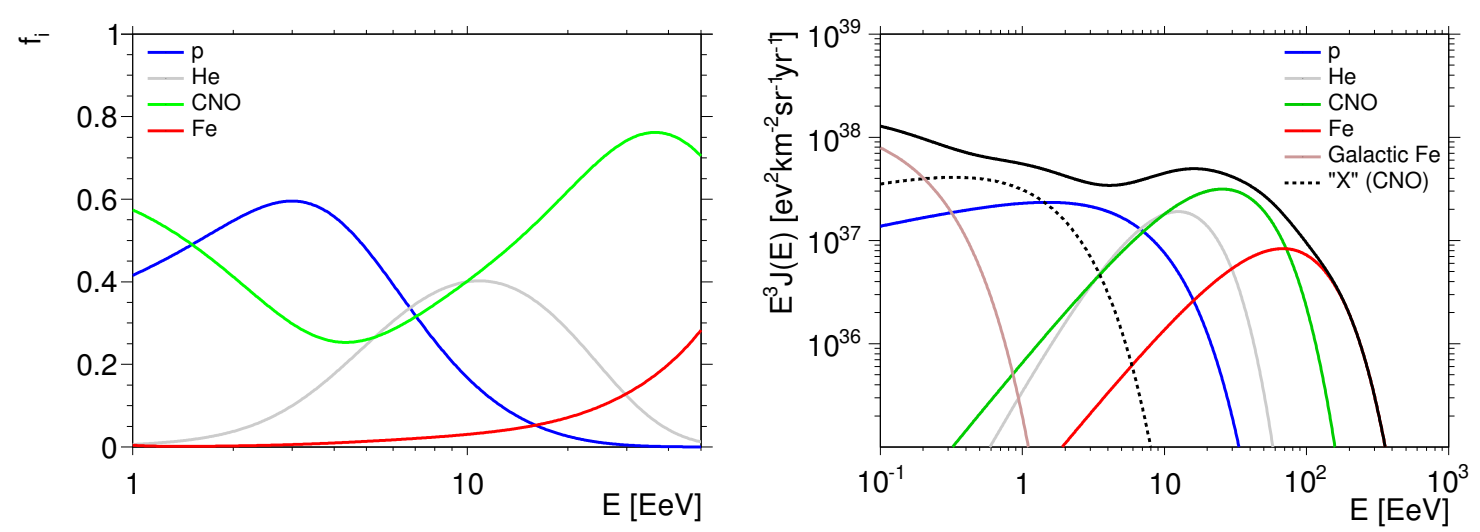

Figure 2: Relative abundances (left) and energy spectrum (right) in the scenario with three components.

is surprising, because such CNO nuclei are not expected to mark the end of the bulk of GCRs in the framework of a rigidity-dependent maximum acceleration energy, such as in the paradigm of supernova remnants as the sources of GCRs. On the other hand, such a fraction of CNO elements in this energy range is not expected either to this extent from the extragalactic scenario because of the much harder spectral indexes required to reproduce the energy spectrum and the relative abundances at higher energies.

Adding this component to the global scenario previously exposed, the obtained relative abundances and all-particle energy spectrum are shown in figure 2. In the energy spectrum, the additional component, referred to as ' $\mathrm{X}(\mathrm{CNO})$ ', is shown by the dotted curve. The energy cut-off for this component is observed to be close to the ankle energy. Overall, this global scenario with three components is seen to reproduce quite well the energy spectrum, and in particular the observed ankle at $5 \times 10^{18} \mathrm{eV}$. On the other hand, the relative abundances are observed to roughly resemble the ones reported in [9] even below the ankle energy. Better agreement could be obtained with more fine-tunings, but given the simplifications of the analysis method already outlined, we consider this scenario as describing sufficiently well the data.

In this framework, it is worth noting that the sharpness of the ankle is explained by the cut-off of the CNO component, and that the ankle marks the transition between this additional component and the extragalactic one. The transition between the bulk of GCRs and extragalactic ones is, on the other hand, taking place progressively above $2 \times 10^{17} \mathrm{eV}$ and is already completed below $10^{18} \mathrm{eV}$. This transition is blurred by the additional CNO component.

The merit of this scenario with three components is to explain the relative abundances observed in the Auger data and the ankle feature in the energy spectrum. However, the obvious new question is to understand the origin of this third component. The last observable at our disposal, namely the angular distribution of the arrival directions of CRs, might offer important keys to face this question.

Large-scale structure of arrival directions. The characterisation of the angular distribution is the third observable which potentially carries important signatures on the origin of CRs. Between the iron-knee and the ankle energies, although with the current sensitivity, the anisotropy ampli- 
tudes are still too small to be measured significantly, some constancy in phase in a direction close to the centre of the Galaxy is observed [18]. This suggests that some fraction of the flux is still of Galactic origin up to $\simeq 10^{18} \mathrm{eV}$. At higher energies, the phase is observed to turn in the direction of the Galactic anti-center, and the signal is strong enough above $8 \times 10^{18} \mathrm{eV}$ to stand out significantly from the background noise [18, 19].

From simple rigidity arguments, the extragalactic flux dominated by protons around and below $\simeq 10^{18} \mathrm{eV}$ could show an anisotropy of the same order as the one of the flux at higher energy progressively dominated by extragalactic $\mathrm{CNO}$ elements above $8 \times 10^{18} \mathrm{eV}$. This should be the case if a significant fraction of the protons observed on Earth are produced in the source environment, or even for secondary protons produced on the way by photo-disintegration processes if the closest sources contributing to the dipole are more distant than the interaction lengths at work. In this case, the extragalactic flux should imprint large-scale anisotropies on the CR flux around $10^{18} \mathrm{eV}$, the size of which should be within the current sensitivity of the Pierre Auger Observatory. However, current measurements are performed without distinction based on the primary mass of CRs. The dipole vector $\mathbf{d}_{\text {tot }}$ to which the upper limits pertain is thus the sum of the vectors $\mathbf{d}_{i}$ associated to each mass group element:

$$
\mathbf{d}_{\mathrm{tot}}(E)=\frac{\sum_{i} \Phi_{i}(E) \mathbf{d}_{i}(E)}{\sum_{i} \Phi_{i}(E)}
$$

where the subscript $i$ runs over all mass group elements and $\Phi_{i}(E)$ is the isotropic part of the corresponding energy spectrum. Hence, a natural mechanism to reduce the amplitude of $\mathbf{d}_{\text {tot }}$ at the level of the observed upper limits relies on non-zero $\mathbf{d}_{i}$ vectors of almost the same amplitude and in opposition of phase. It is to be noted that the phase of extragalactic CRs above $8 \times 10^{18} \mathrm{eV}$ is roughly in the opposite direction to the one for CRs with energies below $10^{18} \mathrm{eV}$ and which is in the general direction of the Galactic Centre. An interesting scenario to explain both upper limits at the percent level around $10^{18} \mathrm{eV}$ and the change of phase around the ankle energy would be that the anisotropy of the $\mathrm{CNO}$ component and of the extragalactic component compensate each other through the opposition of phase of the individual vectors.

Hence, in this scenario, both the $\mathrm{CNO}$ and the proton components would show individually important anisotropies that would almost compensate when considering the sum of the two components due to the opposite directions. The contribution of the end of GCRs through Fe nuclei to both the energy spectrum and the anisotropy would be quite negligible around $10^{18} \mathrm{eV}$.

Outlook. Recent results on the relative abundances above $\simeq 10^{17.8} \mathrm{eV}$, reported in [9], offer important new insights to understand the origin of the ankle feature in the energy spectrum. Unless the paradigm of rigidity-dependent acceleration of CRs in Galactic sources such as supernova remnants is discarded, the presence of a significant fraction of CNO elements combined to the quasi-absence of Fe ones around $10^{18} \mathrm{eV}$ provides evidence for an additional component of CRs. The contribution of this component to the flux of CRs is sub-dominant below the iron-knee energy and negligible above the ankle one.

If the protons observed around $10^{18} \mathrm{eV}$ are of extragalactic origin and follow the same angular distribution as the one of the intermediate nuclei observed above $8 \times 10^{18} \mathrm{eV}$, the angular distribution of the $\mathrm{CNO}$ elements observed around $10^{18} \mathrm{eV}$ should be distributed preferentially from the direction of the Galactic Centre accordingly to a dipolar shape in right ascension with an amplitude 
of the order few percents. This would pinpoint to a Galactic origin for this CNO component. Transient events producing such high-energy CRs in the Galaxy would produce a sharp suppression of the CNO elements at the ankle energy in a quite natural way as the consequence of the escape of the higher energy particles long time ago. The absence of iron nuclei around the ankle energy suggests that the source environment is poor in these elements. This could be natural if the progenitors of these sources are for instance carbon stars. The flux of CRs produced by these events would thus be highly variable in time, and the specific flux detected today on Earth would not be representative of the one that would be observed at other random times. Long gamma-ray bursts could be a candidate for these sources [20].

On the other hand, the anisotropy of extragalactic UHECRs with amplitudes at the few $\%$ level could result from the diffusive propagation in the extragalactic turbulent magnetic field. This could happen if the amplitude of the field is large and/or if the cosmic rays have a component with large electric charge [21]. A large-scale anisotropy is also expected in the case that magnetic deflections are small if the cosmic ray sources are distributed similarly to the matter in the universe, due to the fact that in our local neighbourhood, matter is distributed inhomogeneously.

An important step forward from an observational point of view would be to get the energy spectrum as well as anisotropy measurements for different mass group elements between the iron knee and the ankle energies. Currently, the mass-sensitive observable $X_{\max }$ is measured by telescope fluorescence on an event-by-event basis. The directional exposure of this detection technique is unfortunately very challenging to control with the required accuracy. Hence, it appears of central importance to extract mass-sensitive information on an event-by-event basis with a surface detector array to be able to estimate accurately the corresponding directional exposure. Firm conclusions about the origin of CRs in this energy range could then be drawn.

\section{References}

[1] J. Linsley, Proc. 8th ICRC, vol. 4 p. 77, Jaipur, India (1963)

[2] I. Valiño for the Pierre Auger Collaboration, these proceedings

[3] W. Baade \& F. Zwicky, Proc. National Academy of Sciences of the United States of America 20 (1934) 259

[4] A. R. Bell, Royal Astronomical Society 182 (1978) 443; A. R. Bell, Royal Astronomical Society 182 (1978) 147; R. Blandford \& D. Eichler, Phys. Rep. 154 (1987) 1

[5] E. G. Berezhko \& H. J. Völk, ApJL 661 (2007) L175

[6] W. D. Apel et al. (the KASCADE-Grande Collaboration), Phys. Rev. Lett. 107 (2011) 171104

[7] S. F. Berezhnev et al., Nucl. Instrum. Meth. A 692 (2012) 98

[8] P. Blasi \& E. Amato, JCAP 01 (2011) 010; P. Blasi \& E. Amato, JCAP 01 (2012) 011

[9] The Pierre Auger Collaboration, Phys. Rev. D 90 (2014) 122006

[10] W. D. Apel et al. (the KASCADE-Grande Collaboration), Phys. Rev. D 87 (2013) 081101

[11] A. M. Hillas, J. Phys. G: Nucl. Part. Phys. 31 (2005) R95

[12] T. K. Gaisser, Astropart. Phys. 35 (2012) 801 
[13] T. K. Gaisser, T. Stanev, \& T. Tilav, Front. Phys. 8 (2013) 748

[14] R. Aloisio, V. Berezinsky \& P. Blasi, JCAP 1410 (2014) 020

[15] A. M. Taylor, Astropart. Phys. 54 (2014) 48

[16] A. Di Matteo for the Pierre Auger Collaboration, these proceedings

[17] N. Globus et al., MNRAS 451 (2015) 751

[18] I. Al Samarai for the Pierre Auger Collaboration, these proceedings

[19] The Pierre Auger Collaboration, ApJ 802 (2015) 111

[20] D. Eichler \& M. Pohl, ApJ 711 (2011) 114

[21] D. Harari, S. Mollerach \& E. Roulet, Phys. Rev. D 89 (2014) 123001 